\title{
Estrutura Hidroquímica e Biomassa Planctônica no Norte da Baía de TODOS OS SANTOS, BAHIA, BRASIL.
}

\author{
Paulo de Oliveira MAFALDA JR. ${ }^{1}$ \\ PRISCILA MESQUiTA M. DE SOUZA ${ }^{1}$ \\ EDUARDO MENDES DA SILVA ${ }^{2}$
}

\author{
Instituto de Biologia da UFBA \\ Campus Universitário de Ondina \\ 40210-020 Salvador, Bahia. \\ ${ }^{1}$ Laboratório de Plâncton \\ 1.pomafa@ufba.br \\ ${ }^{2}$ Laboratório de ecotoxicologia
}

\begin{abstract}
RESUMO
Os objetivos deste trabalho foram analisar o padrão de distribuição espacial e sazonal da biomassa fitoplanctônica, microplanctônica e mesoplanctônica, descrever a estrutura hidroquímica da massa d'água e analisar a relação entre os níveis de biomassa planctônica e as características ambientais na Baía de Todos os Santos (Bahia, Brasil). As amostras foram coletadas entre fevereiro/94 e dezembro/95, em 5 estações de amostragem, durante 8 cruzeiros. O fitoplâncton (clorofila a) e o zooplâncton (biomassa de micro e de mesoplâncton) foram coletados através de garrafa de van Dorn e de rede cônica, com malhas de $100 \mu \mathrm{m}$ e $200 \mu \mathrm{m}$ respectivamente. No estudo hidrográfico as seguintes variáveis foram avaliadas: profundidade, temperatura de superfície, salinidade, pH, saturação de oxigênio, transparência, sólidos totais em suspensão, amônia, ortofosfato, silicato, carbono orgânico particulado, fenóis e óleos e graxas. A massa de água tropical presente no norte da Baía de Todos os Santos foi caracterizada como água costeira. Os resultados demonstram que existem diferenças significativas, sazonais e espaciais, principalmente nas variáveis hidroquímicas e secundariamente nos níveis de biomassa planctônica. A análise estatística multivariada revelou 2 agrupamentos entre as estações de amostragem; o grupo 1, formado pelas estações sob influência de atividades de refino (estação 1) e extração de petróleo (estação 4). O grupo 2 formado pelas estações sob influência de atividades de transporte de petróleo (estações 2 e 3) e pela estação referência (estação 5).
\end{abstract}

Palavras chave: Biomassa fitoplanctônica, microfitoplanctônica e mesoplanctônica; hidrologia.

\section{Hydrochemical Structure and Zooplanktonic Biomass in the North of Todos os Santos Bay, Bahia, Brazil}

\begin{abstract}
The objectives of this study were to analyze the pattern of spatial and seasonal distribution of the phytoplanktonic, microplanktonic and mesoplanktonic biomass, to describe the hydrochemical structure of the water and to analyze the relationship between the levels of plankton biomass and environmental variables in the Todos os Santos Bay (Bahia, Brazil). Samples were collected between at February 1994 and December 1995, five sampling stations, during eight cruises. The phytoplankton (chlorophyll a) and the zooplankton (microplankton and mesoplankton
\end{abstract}


biomass) were collected with the help of a bottle van Dorn and a conical net, $100 \mu \mathrm{m}$ e $200 \mu \mathrm{m}$ respectively. Depth, surface temperature, salinity, $\mathrm{pH}$, oxygen saturation, transparency, total solids in suspension, ammonium, orthophosphate, silicate, particulate organic carbon, phenols, oil and grease were analyzed in the water samples. The waters in the Northern region of the Todos os Santos Bay could be characterized as coastal. The results indicate that there were statistically significant differences between stations and seasons, as far as hydrochemical variables are concerned, as well as for planktonic biomass. A multivariate statistics analysis revealed 2 groups between sampling stations, with the first group being formed by the stations influenced mainly by oil refining (station 1) and oil extraction (station 4). The second group was composed by the stations related to oil transportation (stations 2 and 3) and the reference station (station 5).

Key words: Biomassa; chlorophyll; hydrology

\section{INTRODUÇÃO}

A Baía de Todos os Santos (BTS) possui 184 km de extensão costeira da Ponta do Garcez até o Farol de Santo Antônio, sendo caracterizada pela presença de pequenas baías e enseadas, possuindo uma área de $927 \mathrm{~km}^{2}$ e uma profundidade média de $102 \mathrm{~m}$ e onde se encontram mais de 30 ilhas, com cerca de $221 \mathrm{~km}$ de linha de costa (Da Silva, 1994).

O ambiente hidrográfico da BTS abrange três bacias regionais, a saber: a Bacia do Recôncavo Norte, onde destaca-se o Rio Subaé; a Bacia do Paraguaçu, em sua porção leste, representada pelo próprio Rio Paraguaçu e a Bacia do Recôncavo Sul, onde deságua o Rio Jaguaripe, cuja foz situa-se próxima à entrada do Canal de Itaparica, com pouca influência sob o regime hidrológico da Baía de Todos os Santos (CRA, 1982). Não obstante a presença de uma malha hidrográfica considerável, a contribuição de água destes é pequena, pois o rio que antes mais contribuía com considerável volume de água para o interior da baía, o Paraguaçu, com a construção da Barragem Pedra do Cavalo, teve seu fluxo bastante reduzido. Com relação ao movimento de águas na baía, o volume recebido e devolvido durante a maré baixa e maré cheia é de aproximadamente $2,23 \times 10^{9} \mathrm{~m}^{3}$ (DA SILVA, 1994). A velocidade da corrente do Porto de Salvador alcança 0,81 m/s, 3 horas após a preamar (DHN, 1975). Na Ilha de Itaparica, as correntes medidas apresentaram valores mais baixos que não ultrapassaram $0,44 \mathrm{~m} / \mathrm{s}$ na superfície e $0,28 \mathrm{~m} / \mathrm{s}$ no fundo (GUZMAN; NUNES FILHO, 1977).

A Baía de Todos os Santos apresenta o mais elevado índice de densidade demográfica do Estado. Nos dezesseis municípios banhados por suas águas, residem cerca de aproximadamente três milhões de habitantes, que vivem nas cercanias de um parque industrial fundamentado na indústria de exploração e processamento de petróleo, além da indústria de celulose e de extração e beneficiamento de recursos minerais (GERMEN, 1997).

O padrão de precipitação pluviométrica de São Francisco do Conde, local da área de estudo, analisado ao longo de 40 anos, apresentou um período seco, entre setembro e fevereiro e, um período chuvoso entre março e agosto (SEI, 1999). Este padrão também foi verificado na costa norte da Bahia por Mafalda Jr. (2000).

Este estudo foi desenvolvido durante o Programa de monitoramento ambiental ao Norte da Baía de Todos os Santos (Da Silva,1994; Mafalda Jr.,1995), com o objetivo de caracterizar quimicamente a massa d’água, além de avaliar os níveis de produção fito e zooplanctônica, tentando observar possíveis relações com às atividades industriais dominantes, nomeadamente aquelas relacionadas ao petróleo. 


\section{Metodologia}

A área de estudo situa-se ao norte da BTS, onde foram posicionadas quatro estações de amostragem, na região de influência direta das atividades petrolíferas e uma estação referência localizada ao sul da Ilha de Itaparica (Quadro1). A localização dos sítios de amostragem foi realizada através do sistema de navegação por satélite modelo GARMIN-GPS-50.

Quadro 1 - Listagem e localização das estações de amostragem

\begin{tabular}{|c|c|c|}
\hline ESTAÇÕES & $\begin{array}{l}\text { COORDENADAS } \\
\text { GEOGRÁFICAS }\end{array}$ & Atividades \\
\hline 1 - Mataripe & $12^{\circ} 42^{\prime} 06^{\prime \prime} \mathrm{S}$ e $38^{\circ} 34^{\prime} 39^{\prime \prime} \mathrm{W}$ & Refino de Petróleo \\
\hline 2 - Madre de Deus & $12^{\circ} 43^{\prime} 54^{\prime \prime}$ 'S e $38^{\circ} 37^{\prime} 15^{\prime \prime} \mathrm{W}$ & Transporte de Petróleo e derivados \\
\hline $3-$ Pati & $12^{\circ} 42^{\prime} 30^{\prime \prime}$ S e $38^{\circ} 37^{\prime} 30^{\prime \prime} \mathrm{W}$ & Ausência de atividade petrolífera \\
\hline 4 - Ilha das Fontes & 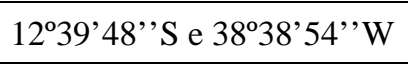 & Exploração de Petróleo \\
\hline 5 - Jiribatuba (referência) & 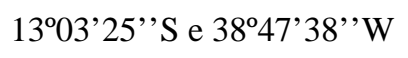 & Ausência de atividade petrolífera \\
\hline
\end{tabular}

A estação 1 localizou-se em um braço de mar, denominado de Rio Mataripe, imediatamente ao lado da Refinaria Landulpho Alves (RLAM), cuja principal atividade industrial é o refino de petróleo. A estação 2 situou-se na porção norte do Terminal Marítimo de Madre de Deus (TEMADRE), responsável pelas atividades de transporte de petróleo e seus derivados. Em frente ao manguezal da Ilha do Pati, localizou-se a estação 3. A estação 4, situou-se em frente a Ilha das Fontes, em cujas adjacências é realizada ainda exploração de petróleo. Situada em Jiribatuba, a estação 5 (referência), caracterizou-se pela presença de manguezal e pela ausência de atividade industrial (Figura 1).

A amostragem hidrográfica e de biomassa planctônica foi realizada em embarcação tipo saveiro durante 2 ciclos de monitoramento, preamar e baixa-mar. No primeiro ciclo foram realizados os cruzeiros: I (fevereiro/94), II (maio/94), III (setembro/94) e IV (dezembro/94), enquanto que, no segundo ciclo foram executados os cruzeiros: V (março/95), VI (junho/95), VII (setembro/95) e VIII (dezembro/95).

A biomassa fitoplanctônica foi determinada através do conteúdo de clorofila $a(\mu \mathrm{g} / \mathrm{L})$, a partir de amostras de água coletadas na superfície, com garrafa tipo van Dorn. Em laboratório procedeu-se a filtração da água, com emprego de bomba de vácuo, ficando o material retido em filtro de membrana de fibra de vidro (Whatmann, GF/C, Ø $47 \mathrm{~mm}$ ). A extração da clorofila empregou como solvente o etanol 90\%. A leitura das densidades óticas dos pigmentos foi realizada através de espectrofotômetro Micronal B382, com valores de absorbância de 750 e 665 m. Após a leitura espectrofotométrica o conteúdo de clorofila $a$ foi calculado pela fórmula de STRICKLAND \& PARSONS (1965).

Para obtenção do zooplâncton, foram realizados arrastos horizontais de superfície com duração de 5 minutos. As redes do tipo cônica, com malha de 100 (microplâncton) e 200 (mesoplâncton) $\mu \mathrm{m}$, foram dotadas de fluxômetro (Hydrobios) para o cálculo do volume de água filtrada. O zooplâncton foi acondicionado em frascos de polietileno e fixado em solução de formalina a $4 \%$. A biomassa de microplâncton e de mesoplâncton $\left(\mathrm{ml} / \mathrm{m}^{3}\right)$ foi estimada a partir da amostra total através do método de deslocamento de biovolume (Kramer et al., 1972). 
No estudo hidroquímico as seguintes variáveis foram monitoradas: profundidade, temperatura, salinidade, saturação de oxigênio, $\mathrm{pH}$, transparência, sólidos totais em suspensão, fenóis, óleos e graxas, amônio, ortofosfato, silicato e carbono orgânico particulado. O monitoramento dos parâmetros realizou-se à superfície e utilizando os valores médios entre a maré alta e maré baixa.

A temperatura e a salinidade foram medidas através de termômetro de inversão e condutivímetro, respectivamente. A transparência da água foi estimada através do disco de Secchi. $\mathrm{O}$ pH foi obtido através do medidor Methron, modelo 025. A saturação de oxigênio foi analisada através do medidor WTW, Mod.OXI-196.

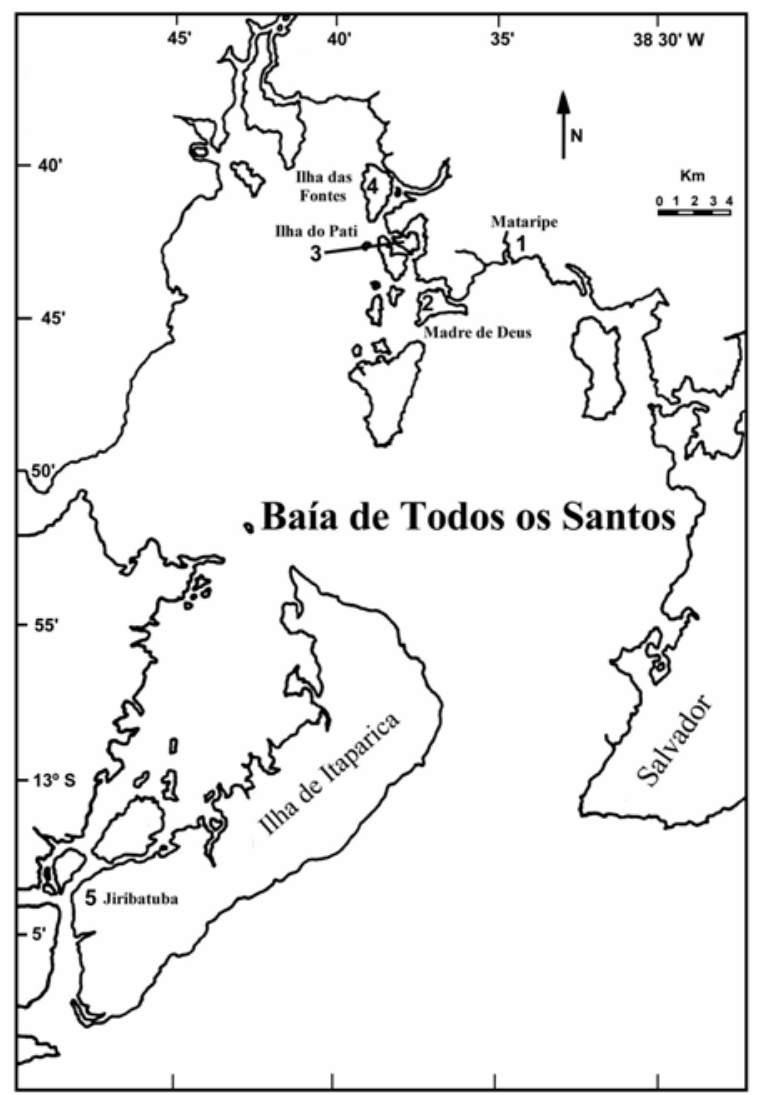

WALLNER-KERSANACH, 1994 (ADAPTADO)

Figura 1 - Mapa de localização das estações de amostragem, no período de Fevereiro/1994 a Dezembro/1995, ao norte da baía de Todos os Santos, Bahia.

As amostras da água para as análises químicas foram coletadas à superfície e ao fundo com o auxílio de uma garrafa van Dorn. Os procedimentos para análises de fenóis, sólidos totais em suspensão, carbono orgânico particulado e óleos e graxas seguiram as recomendações de Apha (1992). Para análise de nutrientes utilizou-se a metodologia contida em Grasshoff (1975). 
O tratamento estatístico multivariado envolveu a Análise de Componentes Principais (PCA). A técnica do PCA foi realizada por intermédio do programa CANOCO versão 2.1 (Ter Braak, 1988), que permite hierarquizar os fatores responsáveis pela variância dos dados e sintetizar as principais tendências através da sua representação gráfica em um número reduzido de planos fatoriais. A matriz formada com os dados oceanográficos foi submetida à padronização para reduzir o efeito das diferentes escalas. Após o cálculo da similaridade por correlação, computou-se os autovalores da matriz de dispersão, que medem a variância associada a cada componente principal. O primeiro eixo descreve a maior dimensão da elipsóide multidimensional, enquanto que os eixos seguintes passam por dimensões gradativamente menores (Legendre \& Legendre, 1984).

\section{RESUltados E Discussão}

\section{Variáveis oceanográficas}

\section{Profundidade}

Durante o primeiro ciclo de monitoramento, a maior amplitude na distribuição da profundidade foi observada em fevereiro/94, oscilando entre 1,6 e 8,1 m, sendo esses os respectivos valores mínimo e máximo da profundidade (Figura 2). O valor médio da profundidade neste período foi de $4,2 \mathrm{~m}$.

No segundo ciclo de monitoramento, o valor mínimo registrado da profundidade foi 2,2 m nos meses de junho e dezembro/95 e o valor máximo foi 6,3 m no mês de setembro/95. A maior amplitude na distribuição da profundidade foi observada também em setembro/95, com valores entre 2,3 e 6,3 m. Neste período, o nível médio da profundidade foi de 3,4 m.

Apesar da amplitude verificada na profundidade das estações de amostragem a diferença entre os valores médios não foi significativa entre os cruzeiros (ANOVA, $p=0,9231$ ). As estações 1 e 4 apresentaram menores profundidades em relação as estações 2, 3 e 5 (ANOVA, p = 0,002).

\section{Temperatura}

No período de fevereiro/94 a dezembro/94 a temperatura variou espacial e temporalmente, com média de $26,6{ }^{\circ} \mathrm{C}$. O valor mínimo foi encontrado em setembro/94 $\left(22,0{ }^{\circ} \mathrm{C}\right)$ e o máximo ocorreu em fevereiro/94 (30,9 ${ }^{\circ} \mathrm{C}$ ) (Figura 2). No mês de fevereiro/94 constatou-se a maior amplitude na distribuição espacial da temperatura, oscilando entre 22,9 e 30,9 ${ }^{\circ} \mathrm{C}$.

Entre março/95 e dezembro/95, o valor máximo foi registrado em dezembro/95 $\left(29,0^{\circ} \mathrm{C}\right)$ e o mínimo em setembro/95 $\left(25,9^{\circ} \mathrm{C}\right)$; Neste mesmo mês foi confirmada a maior amplitude $(25,9$ e $27,7^{\circ} \mathrm{C}$ ). A média encontrada nesse período foi de $27,4^{\circ} \mathrm{C}$.

A análise de variância indicou uma diferença significativa da temperatura entre os cruzeiros estudados (ANOVA, p = 0,0077), indicando a variação temporal da temperatura. Posteriormente, o teste de comparações múltiplas de Dunn, verificou que esta diferença ocorreu apenas entre os cruzeiros de setembro/94 e dezembro/95.

A sazonalidade térmica, em ambos os ciclos, foi caracterizada por temperaturas mais elevadas nos meses menos chuvosos e temperaturas mais baixas nos meses de mais chuvosos. Este padrão térmico também foi descrito para a área adjacente na costa norte do Estado da Bahia por Mafalda Jr.(2000).

A temperatura da água nas estações amostradas apresentou diferença significativa (ANOVA, p = 0,05) entre a estação 1 e as demais, inclusive a referência, provavelmente em função da cor da água que na estação 1 é mais escura devido a presença de substâncias húmicas decorrentes 
do processo de decomposição da lignina de vegetais, esta absorvem majoritariamente os raios infravermelhos aumentando sobremaneira a temperatura (Wetzel, 1983).

\section{Salinidade}

No primeiro ciclo de estudo, o valor máximo foi registrado em maio/94 (36,0 UPS) e o mínimo em setembro/94 (30,3 UPS), neste mesmo mês foi registrada a maior amplitude na distribuição espacial da salinidade que variou entre 30,3 e 34,4 UPS (Figura 2). A salinidade média da água foi de 33,8 UPS.

No segundo ciclo de estudo, a salinidade média foi de 33,2 UPS. A maior amplitude foi observada em março/95 (28,0 e 34,6 UPS), sendo 28,0 UPS o valor mínimo e 35,1 UPS o valor máximo encontrado em junho/95.

A variação temporal da salinidade foi constatada através da diferença muito significativa (ANOVA, p=0,0024) durante o período de estudo. O teste de comparações múltiplas de Dunn constatou que esta diferença aconteceu entre os cruzeiros de fevereiro/94 e setembro/94, e fevereiro/94 e dezembro/95.

\section{Diagrama T/S}

A massa de água costeira presente ao norte da Baía de Todos Santos pode ser visualizada através dos diagramas T-S (Figura 3). A amplitude térmica $\left(22,0-30,9^{\circ} \mathrm{C}\right)$ e salina $(30,3-36,0$ UPS) encontrada durante o primeiro ciclo de monitoramento foi mais elevada do que a amplitude térmica $\left(25,9-29,0^{\circ} \mathrm{C}\right)$ e salina (28,0-35,1 UPS) obtida no segundo ciclo. As diferenças verificadas na amplitude da salinidade entre os dois ciclos de monitoramento podem ser atribuídas a diferenças na taxa de pluviosidade e conseqüente modificação da vazão estuarina.

Segundo Thonsen (1962), a água costeira que cobre a plataforma continental é essencialmente água oceânica com mistura de água dos rios. Contudo, mesmo nos períodos de menor pluviosidade, não foi verificada a ocorrência da massa de água oceânica como aquela caracterizada na costa norte da Bahia durante o período seco (Mafalda Jr., 2000), refletindo, desta forma, o efeito dos aportes fluviais na formação da água costeira do norte da Baía de Todos os Santos. A massa de água oceânica, que é característica da Corrente do Brasil, é caracterizada por temperatura superior a $18^{\circ} \mathrm{C}$ e salinidade maior que 36 UPS (Garfiled, 1990). 

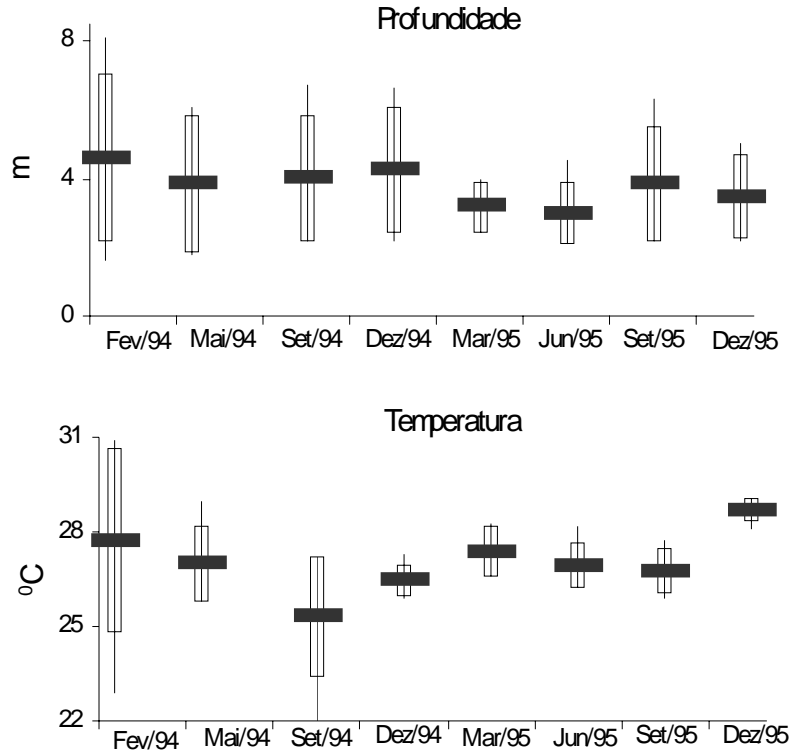

\section{Salinidde}

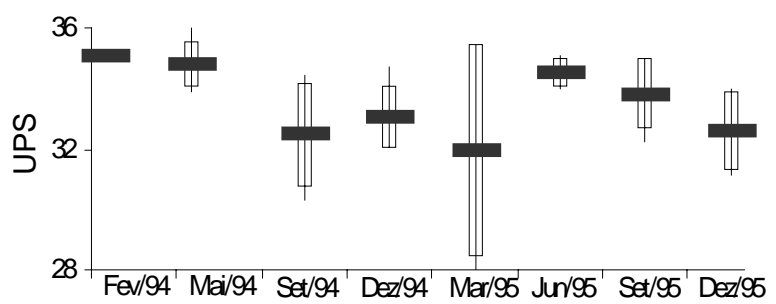

Figura 2 - Variação sazonal dos valores médios de profundidade $(\mathrm{m})$, temperatura $\left({ }^{0} \mathrm{C}\right)$ e salinidade (UPS), no período de fevereiro/94 a dezembro/95, ao norte da Baía de Todos os Santos (valores máximo e mínimo: linha vertical; média: barra horizontal; desvio-padrão: retângulo). 
Primeiro ciclo

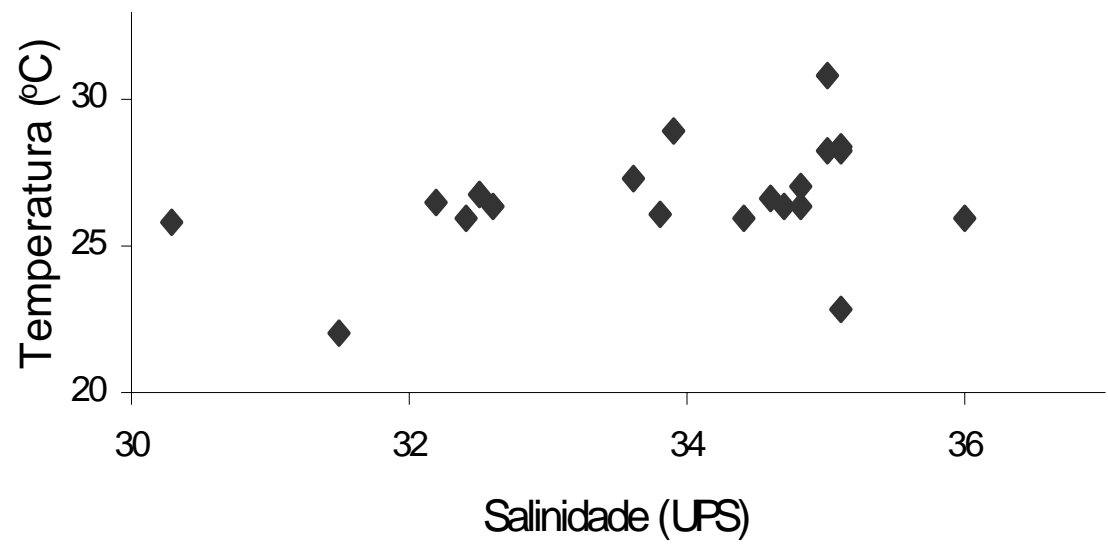

Segundo ciclo

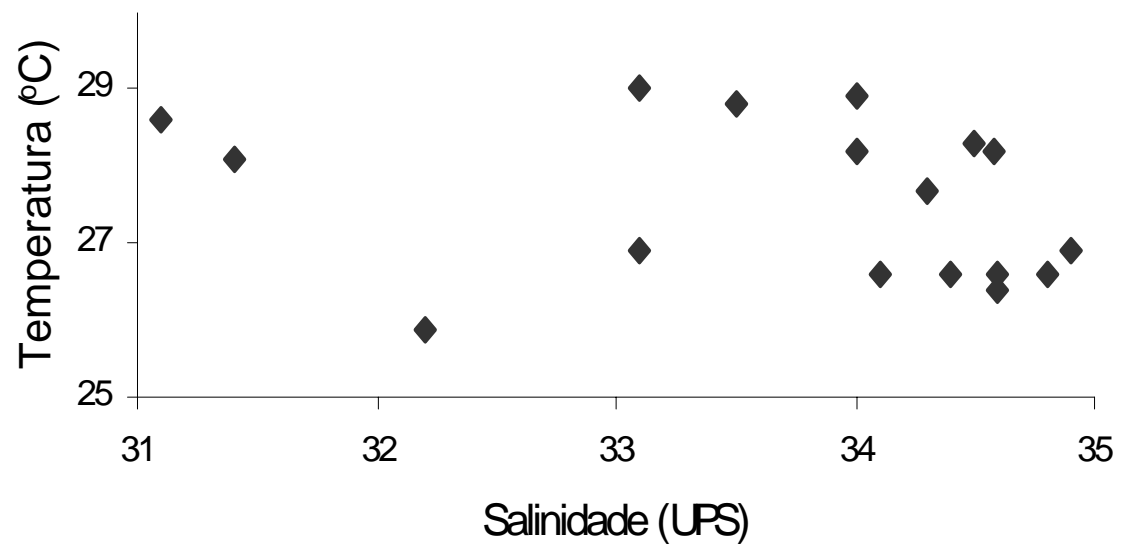

Figura 3 - Caracterização das massas de água, através do diagrama T/S, nos períodos de fevereiro/1994 a dezembro/1994 (primeiro ciclo) e março/1995 a dezembro/1995 (segundo ciclo), ao norte Baía de Todos os Santos.

Tropical Oceanography, Recife: v. 31, n. 1, p. 31-51, 2003 


\section{Saturação de Oxigênio}

No primeiro ciclo, a maior amplitude na distribuição espacial da saturação de oxigênio ocorreu em maio/94, cujos valores oscilaram entre 79,8 e 104,8 \% (Figura 4). O nível médio de saturação de oxigênio foi de 91,3\%. Em fevereiro/94 foi encontrado o valor mínimo (73,0 \%) e em maio/94 foi registrado o valor máximo (104,8 \%).

No segundo ciclo, o nível médio da saturação de oxigênio foi de 92,5\%. No mês de setembro/95 foram registrados os valores máximo e mínimo da saturação de oxigênio e a maior amplitude, cujos valores oscilaram entre 75,7 e 107,3\%.

A variação nos valores de saturação de oxigênio foi confirmada através do teste ANOVA, com p= 0,0023, apontando uma diferença muito significativa entre os valores obtidos. No teste de Dunn verificou-se diferença entre fevereiro/94 e dezembro/94, e entre fevereiro/94 e junho/95.

Em ambos os ciclos os valores de oxigênio estiveram próximos ao ponto de saturação de 100 \%, assim como verificado na costa norte da Bahia (Mafalda Jr., 2000).

\section{Transparência}

No primeiro ciclo, o valor médio da transparência foi de $1,5 \mathrm{~m}$. A maior amplitude foi encontrada em dezembro/94, com valores entre 0,7 e $2,8 \mathrm{~m}$ (Figura 4). O valor máximo foi registrado em setembro e dezembro/94 (2,8 m) e o mínimo em dezembro/94 (0,7 m).

No segundo ciclo foi registrado em março/95 o valor mínimo da transparência $(0,5 \mathrm{~m})$ e em dezembro/95 foi constatado o valor máximo (2,5 m). O nível médio da profundidade foi de 1,5 m. As maiores amplitudes foram encontradas nos meses de junho, setembro e dezembro/95, cujos valores respectivos foram: (0,9 e 2,3 m), (1,0 e 2,4 m) e (1,1 e 2,5 m).

O teste não-paramétrico de Kruskal-Wallis constatou que não houve diferença significativa entre os valores de transparência (ANOVA, $\mathrm{p}=0,7613$ ).

Foi encontrada diferença muito significativa (ANOVA $=0,001$ ) entre os valores de transparência que foram menores nas estações 1 e 4, em relação as estações 2, 3 e referência (5).

\section{pH}

No primeiro ciclo foi constatado a maior amplitude do $\mathrm{pH}$ em dezembro/94, com valores entre 7,6 e 8,1, nesse mesmo mês foi registrado o valor mínimo $(7,6)$, enquanto o valor máximo foi encontrado em setembro/94.

No segundo ciclo, no mês de setembro/95 registrou-se a maior amplitude do $\mathrm{pH}$, que oscilou entre 6,8 e 7,5 (Figura 4). O valor médio do $\mathrm{pH}$ foi 7,6. Em setembro/95 foi constatado o valor mínimo de $\mathrm{pH}(6,8)$, enquanto o máximo $(8,0)$ foi registrado em março, junho e dezembro/95. A variação dos valores de $\mathrm{pH}$ foi confirmada através da diferença extremamente significativa (ANOVA $\mathrm{p}=0,0007$ ) entre os cruzeiros. No teste de Dunn constatou-se a diferença nos meses de fevereiro/94 e setembro/95, e setembro/94 e setembro/95.

O pH da água oceânica varia entre 8,0 e 8,3 (Margalef, 1989), contudo em áreas costeiras a água do mar pode apresentar um pH maior ou menor do que 7,0 (Friedrich, 1969), refletindo o efeito do aporte continental.

\section{Sólidos Totais em Suspensão}

No primeiro ciclo, o nível médio dos sólidos foi de 24,8 mg/L e a maior amplitude ocorreu em setembro/94, cujos valores oscilaram entre 13,6 e 47,8 mg/L (Figura 4), sendo este último o valor máximo registrado, enquanto o mínimo (5,1 mg/L) ocorreu em dezembro/94. 
No segundo ciclo, a maior amplitude ocorreu em março/95, com valores oscilando entre 6,7 e $25 \mathrm{mg} / \mathrm{L}$, sendo esse último o valor máximo registrado, enquanto o mínimo foi encontrado em junho/95 (2,9 mg/L). O valor médio dos sólidos totais em suspensão foi de 9,7mg/L.

A análise de variância não-paramétrica indicou uma diferença extremamente significativa dos sólidos durante o período de estudo (ANOVA, p=0,0004). Através do teste de Dunn, verificouse a diferença nos meses de maio/94 e junho/95, maio/94 e setembro/95, e maio/94 e dezembro/95. Assim como para a profundidade e transparência, também foi encontrada diferença significativa (ANOVA $=0,01$ ) entre os valores de sólidos em suspensão das estações 1 e 4 , em relação as estações 2, 3 e referência (5). Ou seja, as estações que apresentaram as maiores concentrações de sólidos em suspensão foram as que apresentaram as menores profundidades e como conseqüência a menor transparência em função do maior revolvimento do fundo que pode provocar a ressuspensão das partículas orgânicas e inorgânicas na coluna de água.
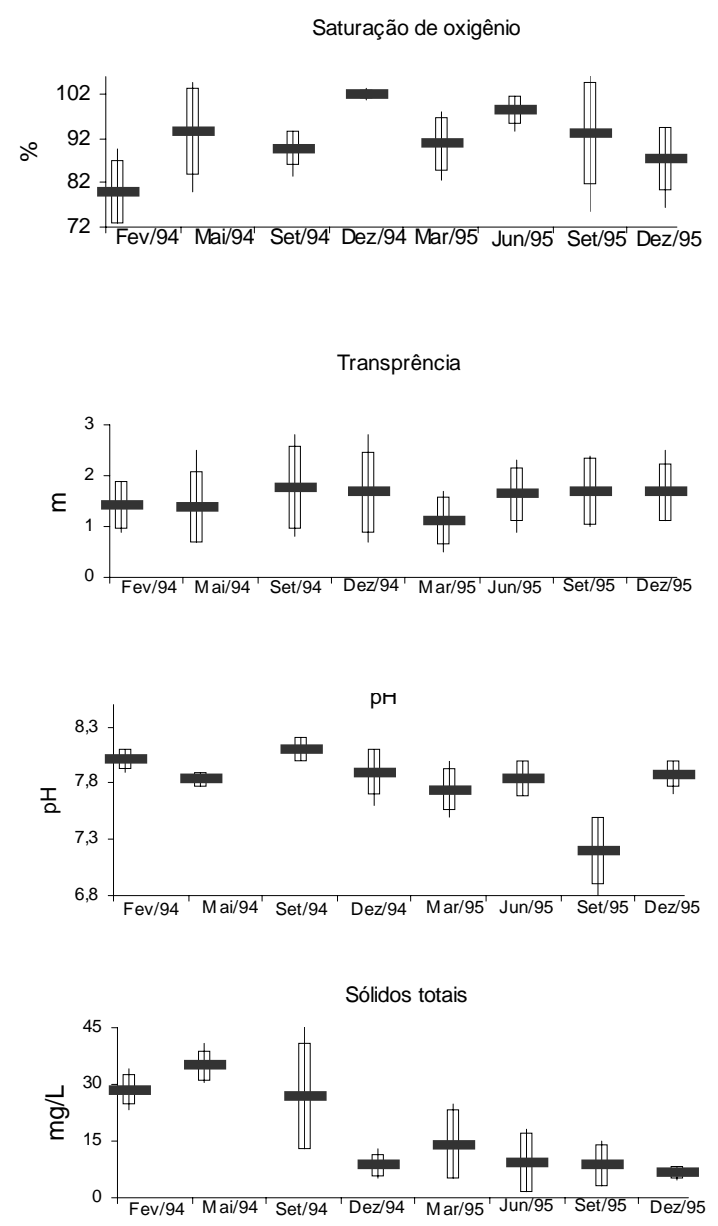

Figura 4 - Variaçao sazonaı aos vaıores meaıs aa saturaçao ae oxıgenıo (\%), transparencıa (m), pH e sólidos totais (mg/L), no período de fevereiro/1994 a dezembro/1995, ao norte da Baía de Todos os Santos (valores máximo e mínimo: linha vertical; média: barra horizontal; desvio-padrão: retângulo). 


\section{Fenóis}

Durante o primeiro ciclo, o nível médio de fenóis foi de 22, $\mu \mathrm{g} / \mathrm{L}$ e o valor máximo (36,1 g/L) ocorreu em maio/94 e o mínimo (11,3 $\mu \mathrm{g} / \mathrm{L})$ em dezembro/94. Nesse mesmo mês foi constatado a maior amplitude, cujos valores oscilaram entre 11,3 e 32,9 $\mu \mathrm{g} / \mathrm{l}$.

No segundo ciclo, a maior amplitude ocorreu em dezembro/95, cujos valores variaram entre 23,7 $3119 \mu \mathrm{g} / \mathrm{L}$ (Figura 5), sendo este último o valor máximo encontrado, enquanto que o mínimo ocorreu em junho/95 (7,6 $\mu \mathrm{g} / \mathrm{L})$. O nível médio de fenóis foi de 21,7 $\mu \mathrm{g} / \mathrm{L}$.

O teste não paramétrico demonstrou uma diferença muito significativa entre os valores de fenóis (ANOVA, p=0,0015). Através do teste de comparação múltipla de Dunn, constatou-se esta diferença nos meses de maio/94 e junho/95, e entre junho/95 e dezembro/95.

Verificou-se diferença significativa entre a estação 1 e as estações 2, 3 e referência (5) mas não entre a estação 1 e estação 4 (ANOVA, p=0,02). Estes resultados apontam a influência das atividades de refino (estação 1) e de extração (estação4) de petgróleo sobre a queda da qualidade da água ao norte da Baía de Todos os Santos. A EPA (Agência Estado-Unidense de Proteção Ambiental) estabelece que a concentração máxima de compostos fenólicos permitida para a manutenção dos processos biológicos em corpos aquáticos é de $100 \mu \mathrm{g} / \mathrm{L}$ e para o estabelecimento de $\mathrm{LC}_{50}-96 \mathrm{~h}$ e de 5,0 $\mu \mathrm{g} / \mathrm{L}$. Em alguns momentos, os valores encontrados na área de estudo chegam a se superiores aos recomendados pela EPA

\section{Óleos e Graxas}

No primeiro ciclo, o valor mínimo foi registrado em fevereiro/94 (1,4 mg/L) e o máximo em dezembro/94 (27,0 mg/L), nesse mesmo mês ocorreu a maior amplitude, cujos valores oscilaram entre 15,7 e 27,0 mg/L (Figura 5). O valor médio de óleos e graxas foi de 7,7 mg/L.

A maior amplitude foi encontrada no segundo ciclo, em dezembro/95, com valores oscilando entre 4,7 e 52,6 mg/L, sendo esse último o valor máximo registrado, enquanto o mínimo foi encontrado em setembro/95 (3,4 mg/L). Durante este período, o nível médio de óleos e graxas foi de $17,3 \mathrm{mg} / \mathrm{L}$.

A ANOVA não-paramétrica encontrou diferença extremamente significativa entre os valores analisados (ANOVA, $\mathrm{p}=0,0001$ ). $\mathrm{O}$ teste de Dunn constatou a diferença entre os meses de fevereiro/94 e dezembro/94, fevereiro/94 e março/95, fevereiro/94 e dezembro/95, maio/94 e dezembro/94 e maio/94 e dezembro/95.

Não foi detectada a existência de diferença significativa entre as estações de amostragem (ANOVA, p =0,812). Segundo a APHA (1992) óleos e graxas é uma fração composta primariamente por matéria graxa de origem animal e vegetal e por hidrocarbonetos de origem petrolífera. Contudo a metodologia de análise empregada não permitiu diferenciar os distintos componentes desta fração e desta forma não foi possível determinar a extensão da influência da RLAM, com relação a esta variável. 

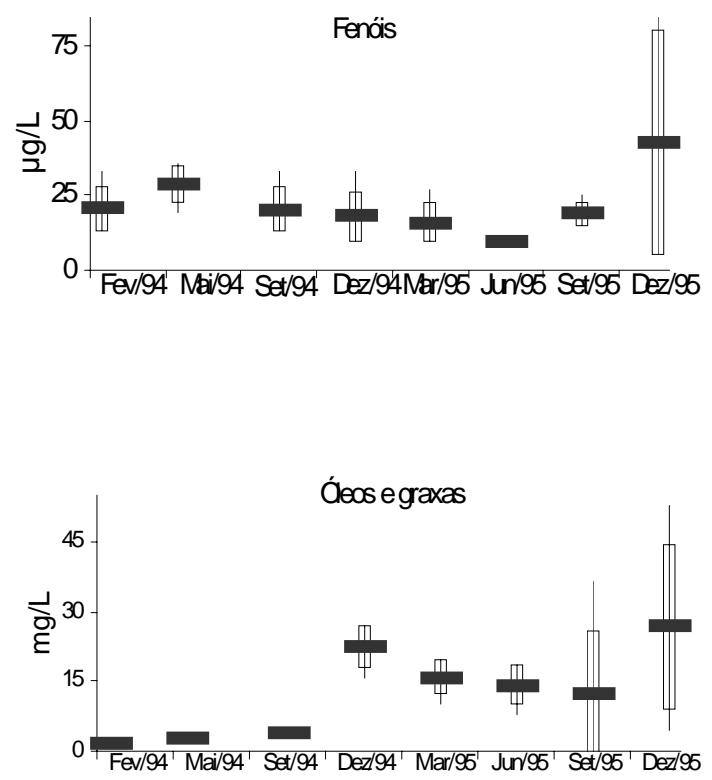

Figura 5 - Variação sazonal dos valores médios de fenóis ( $\mu \mathrm{g} / \mathrm{L})$ e óleos e graxas $(\mathrm{mg} / \mathrm{L})$, no período de fevereiro/1994 a dezembro/1995, ao norte da Baía de Todos os Santos (valores máximo e mínimo: linha vertical; média: barra horizontal; desvio-padrão: retângulo).

\section{Amônio $\left(\mathrm{NH}_{4}^{+}\right)$}

Durante o primeiro ciclo foi registrada a maior amplitude (0,6 e 9,0 $\mu \mathrm{M})$ em fevereiro/94, sendo esse último o valor máximo encontrado, enquanto o valor mínimo ocorreu em setembro/94 $(0,3 \mu \mathrm{M})$. O nível médio do amônio foi de $2,1 \mu \mathrm{M}$.

Segundo ciclo - O valor médio do amônio foi de 2,6 $\mu \mathrm{M}$. Em setembro/95 registrou-se a maior amplitude dos valores de amônio, entre 1,2 e 10,5 $\mu \mathrm{M}$ (Figura 6), sendo este último o valor máximo encontrado, enquanto o mínimo ocorreu em março/95 (0,9 $\mu \mathrm{M})$.

O teste não-paramétrico verificou que a diferença entre os valores de amônio obtido nos 8 cruzeiros não foi significativa (ANOVA, $p=0,1692$ ). Foi encontrada diferença muito significativa (ANOVA, $p=0,001$ ) entre as estações 1 e 4 e a estação referência (5). Os valores muito elevados da estação 1 são conseqüência da contribuição oriunda dos efluentes da RLAM. Contudo, a drenagem terrestre e a remoção de nutrientes a partir do sedimento podem provocar a eutrofização em regiões costeiras, modificando o padrão esperado para águas tropicais (Tundisi et al.,1978).

\section{Ortofosfato}

No primeiro ciclo, o valor máximo de ortofosfato foi registrado em dezembro/94 (1,2 mg/L) e o mínimo (0,3 mg/L) ocorreu em setembro/94 (Figura 6). A concentração média de ortofosfato foi de $0,7 \mathrm{mg} / \mathrm{L}$. A maior amplitude na distribuição espacial dos valores médios de ortofosfato foi em dezembro/94, com variação entre 0,5 e $1,2 \mathrm{mg} / \mathrm{L}$ 
O segundo ciclo caracterizou-se pela maior amplitude em setembro/95, cujos valores oscilaram entre 0,2 e 1,0 mg/L. O valor máximo de 1,0 mg/L foi registrado nos meses de março, junho e setembro/95, enquanto o valor mínimo $(0,2 \mathrm{mg} / \mathrm{L})$ foi constatado em setembro e dezembro/95.

A análise estatística não-paramétrica indicou uma diferença extremamente significativa nos valores de ortofosfato (ANOVA, $\mathrm{p}=0,0018$ ) entre os cruzeiros estudados. O teste de Dunn verificou que esta diferença ocorreu entre os meses de fevereiro/94 e dezembro/95 e, entre dezembro/94 e dezembro/95. Não houve diferença significativa nos valores entre as estações de amostragem $($ ANOVA $=0,462)$.

Na costa atlântica os valores de fosfato inorgânico oscilam entre 0,003 e 0,01 mg/l (Margalef,1989), assim os valores encontrados no norte da Baía de Todos os Santos foram superiores aos esperados para um ambiente costeiro tropical.

\section{Silicatos}

O valor médio de silicato foi de 7,9 mg/L no primeiro ciclo e o valor mínimo (3,2 mg/L) ocorreu em maio/94 e o máximo $(12,4 \mathrm{mg} / \mathrm{L})$ em dezembro/94, nesse mesmo mês constatou-se a maior amplitude, cujos valores foram entre 7,2 e 12,4 mg/L (Figura 6).

No segundo ciclo, o valor mínimo foi verificado em dezembro/95 (0,4 mg/L) e o máximo em março/95 (18,0 mg/L). A maior amplitude nos valores de silicato ocorreu em setembro/95, oscilando entre 2,2 e 12,7 mg/L. O valor médio de silicato foi de $6,8 \mathrm{mg} / \mathrm{L}$.

O teste não-paramétrico constatou uma diferença muito significativa nos valores de silicato (ANOVA, $\mathrm{p}=0,0016$ ), durante o período de estudo. Através do teste de comparações múltiplas de Dunn, verificou-se diferença entre os meses de fevereiro/94 e dezembro/95, e entre março/95 e dezembro/95.

Apesar de não haver diferença significativa nos valores de silicato entre as estações de amostragem (ANOVA = 0,760), as estações 1 e 4 com maior influência terrígena apresentaram os valores médios mais elevados.

\section{Carbono Orgânico Particulado}

Durante o primeiro ciclo ocorreu a maior amplitude, em maio/94, com valores entre $1001 \mathrm{e}$ $2235 \mathrm{mg} / \mathrm{L}$. Nesse mesmo mês foi encontrado o valor máximo (2235 mg/L), enquanto o mínimo (677 mg/L) ocorreu no mês dezembro/94. O valor médio do carbono foi de $1370 \mathrm{mg} / \mathrm{L}$.

No segundo ciclo, a maior amplitude ocorreu em março/95, com variação entre 1307 e $4181 \mathrm{mg} / \mathrm{L}$ (Figura 6). Em setembro/95 foi registrado o valor mínimo (1121 mg/L) e em junho/95 foi apontado o valor máximo (4199 mg/L). O valor médio de carbono foi de $1906 \mathrm{mg} / \mathrm{L}$.

$\mathrm{O}$ teste não-paramétrico constatou uma diferença muito significativa entre os cruzeiros estudados (ANOVA, $\mathrm{p}=0,0021$ ). Posteriormente, através do teste de Dunn verificou-se que esta diferença ocorreu entre os meses de fevereiro/94 e dezembro/94, dezembro/94 e março/95, e dezembro/94 e junho/95.

Foi encontrada diferença significativa (ANOVA, p = 0,04) entre a estação 4 e as estações 2, 3 e referência (5), em função da composição essencialmente orgânica do petróleo, que é extraído nesta região. 

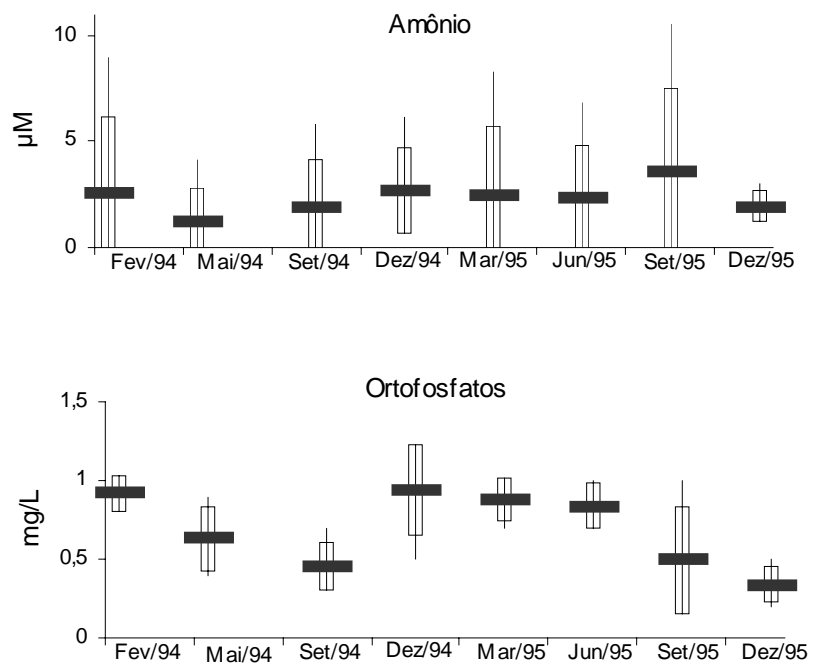

Silicatos
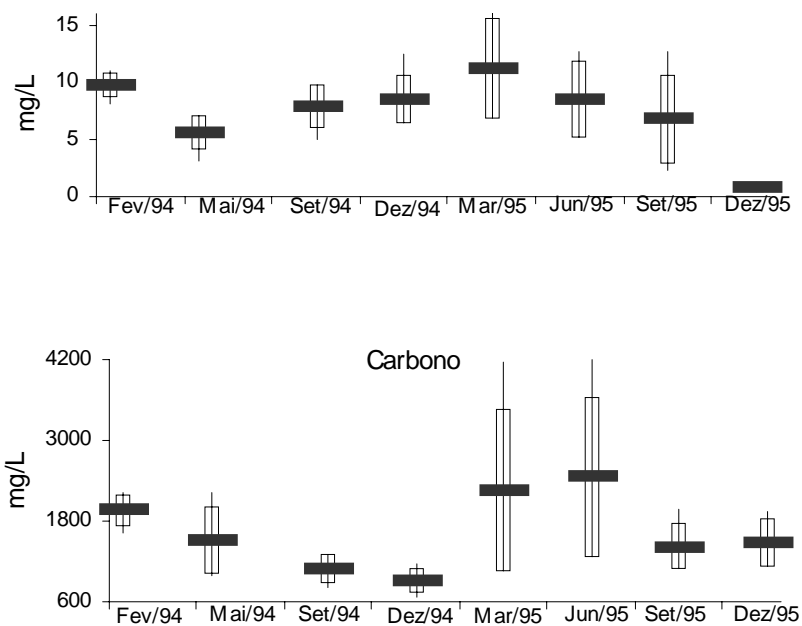

Figura 6 - Variação sazonal dos valores médios de amônio $(\mu \mathrm{M})$, ortofosfatos $(\mathrm{mg} / \mathrm{L})$, silicatos $(\mathrm{mg} / \mathrm{L})$ e carbono (mg/L, no período de fevereiro/1994 a dezembro/1995, ao norte da Baía de Todos os Santos (valores máximo e mínimo: linha vertical; média: barra horizontal; desvio-padrão: retângulo). 


\section{Clorofila $a$}

No primeiro ano de amostragem, o valor mínimo da biomassa fitoplanctônica $(0,1 \mu \mathrm{g} / \mathrm{L})$, medida através da concentração da clorofila, foi encontrado em fevereiro/94 e o máximo $(4,0 \mu \mathrm{g} / \mathrm{L})$ foi em setembro/94. Em dezembro/94, foi registrada a maior amplitude, cujos valores foram entre 1,0 e 3,8 $\mu \mathrm{g} / \mathrm{L}$. A média dos valores da biomassa foi de 3,7 $\mu \mathrm{g} / \mathrm{L}$.

Durante o segundo ciclo, a biomassa fitoplanctônica média foi de 2,8 $\mu \mathrm{g} / \mathrm{L}$. O mês de setembro/95 apresentou a maior amplitude na distribuição espacial (1,5 e 8,7 $\mu \mathrm{g} / \mathrm{L})$, sendo este último, o valor máximo encontrado (Figura 7). O mês de junho/95 apresentou o valor mínimo (0,6 $\mu \mathrm{g} / \mathrm{L})$.

Os valores encontrados para os dois ciclos, quando comparados através da ANOVA apresentaram uma diferença significativa (ANOVA, $p=0,004$ ). Foi encontrada diferença significativa (ANOVA $=0,05$ ) nos valores de clorofila que foram maiores na estação 1 , em relação as estações 2, 3, 4 e referência (5). A clorofila é um dos indicadores da biomassa fitoplanctônica e, por conseguinte importante na determinação do estado trófico do ecossistema (Margalef,1989). Provavelmente, a contribuição nutricional dos efluentes da RLAM, notadamente no que se refere ao amônio, deve estar estimulando o aumento da produção fitoplanctônica, uma vez que, os valores encontrados na costa norte da Bahia, entre 0,01 e 0,35 $\mu \mathrm{g} / \mathrm{L}$ (Mafalda Jr.,2000), são sensivelmente menores aos verificados no norte da Baía de Todos os Santos.

\section{Biomassa de Microplâncton}

A maior amplitude na distribuição espacial dos valores de biomassa microplanctônica ocorreu em setembro/94, no primeiro ano de amostragem, oscilando entre 1,1 e 6,2 $\mathrm{ml} / \mathrm{m}^{3}$ (Figura 7). O nível médio da biomassa foi de $3,7 \mathrm{ml} / \mathrm{m}^{3}$. No mês de fevereiro/94 foi encontrado o valor mínimo $\left(0,4 \mathrm{ml} / \mathrm{m}^{3}\right)$ e o valor máximo $\left(8,7 \mathrm{ml} / \mathrm{m}^{3}\right)$ foi registrado em dezembro/94.

Por outro lado, o segundo ciclo apresentou uma biomassa microplanctônica média de 2,7 $\mathrm{ml} / \mathrm{m}^{3}$. O valor máximo $\left(7,0 \mathrm{ml} / \mathrm{m}^{3}\right)$ foi registrado em setembro/95 e o mínimo $\left(0,2 \mathrm{ml} / \mathrm{m}^{3}\right)$ no cruzeiro de dezembro/95. O nível médio da biomassa foi de $2,7 \mathrm{ml} / \mathrm{m}^{3}$.

O teste não-paramétrico dos valores de biomassa do microplâncton indicou diferença extremamente significativa (ANOVA, $\mathrm{p}=0,0004$ ) entre os cruzeiros. O teste de Dunn realizado posteriormente verificou que esta diferença ocorreu entre os cruzeiros de fevereiro/94 e dezembro/94, e entre dezembro/94 e dezembro/95.

\section{Biomassa do Mesoplâncton}

No primeiro ciclo, o valor mínimo da biomassa mesoplanctônica $\left(0,2 \mathrm{ml} / \mathrm{m}^{3}\right)$ foi encontrado em fevereiro e dezembro/94 e o valor máximo $\left(2,4 \mathrm{ml} / \mathrm{m}^{3}\right)$ em setembro $/ 94$, nesse mesmo cruzeiro foi constatado a maior amplitude, com valores entre 0,4 e $2,4 \mathrm{ml} / \mathrm{m}^{3}$. O nível médio da biomassa mesoplanctônica foi de $0,9 \mathrm{ml} / \mathrm{m}^{3}$.

Durante o segundo ciclo, o valor mínimo da biomassa mesoplanctônica $\left(0,1 \mathrm{ml} / \mathrm{m}^{3}\right)$ foi registrado em março/95 e o valor máximo $\left(4,5 \mathrm{ml} / \mathrm{m}^{3}\right)$ em dezembro/95, nesse mesmo cruzeiro foi constatado a maior amplitude da biomassa, com variação entre $\left(0,3 \mathrm{a} 4,5 \mathrm{ml} / \mathrm{m}^{3}\right)$. A biomassa média foi de $1,7 \mathrm{ml} / \mathrm{m}^{3}$ (Figura 7).

A análise não-paramétrica de Kruskal-Wallis considerou totalmente não significativa a diferença entre os cruzeiros estudados (ANOVA, $p=0,0739$ ). 
Na costa norte da Bahia, os valores da biomassa de micro e de mesoplâncton obtidos no estudo de Mafalda Jr.,2000 foram bastante inferiores aos encontrados no presente estudo, indicando a influência do aporte fluvial e das atividades petrolíferas sobre a biomassa planctônica.

Corofila a

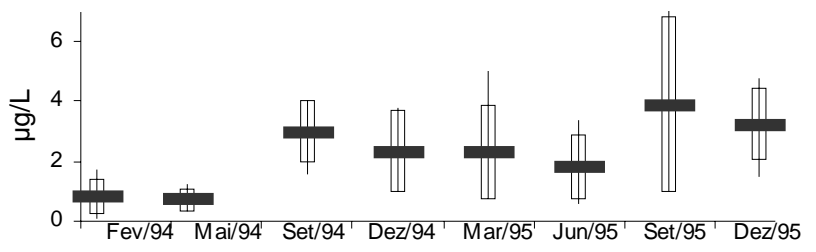

Microzooplâncton

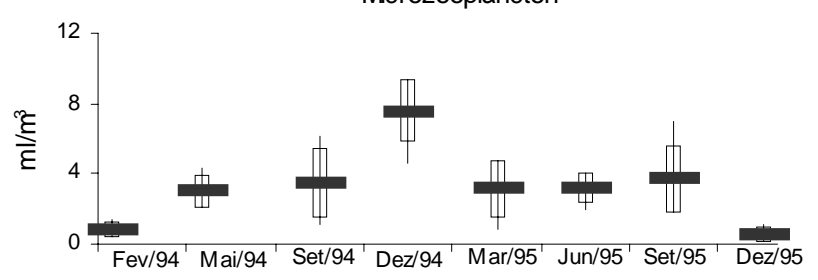

Mesozooplâncton

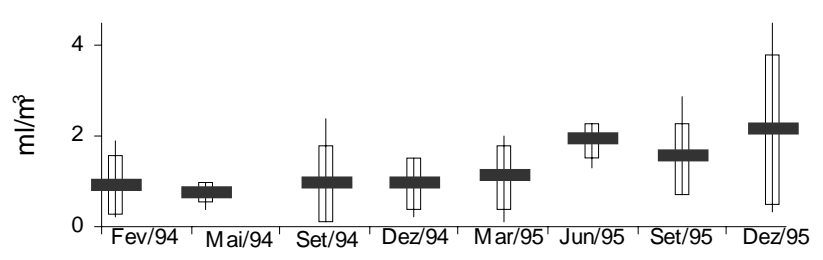

Figura 7 - Variação sazonal dos valores médios de clorofila $a(\mu \mathrm{g} / \mathrm{L})$, microzooplâncton $\left(\mathrm{ml} / \mathrm{m}^{3}\right)$ e mesozooplâncton $\left(\mathrm{ml} / \mathrm{m}^{3}\right)$, no período de fevereiro/1994 a dezembro/1995, ao norte da Baía de Todos os Santos (valores máximo e mínimo: linha vertical; média: barra horizontal; desvio-padrão: retângulo).

\section{Análise de Componentes Principais (ACP)}

As Tabelas 1 e 2 apresentam o autovalor, a percentagem da variação total e a percentagem acumulada dos quatro primeiros componentes principais, que no ciclo 1 explicam 71 \% da variância dos dados oceanográficos, enquanto no ciclo 2, explicam 69,2 \%. 
Tabela 1 - Autovalor, percentagem da variação total e percentagem acumulada dos quatro primeiros componentes principais, no primeiro ciclo de monitoramento no norte da Baía de Todos os Santos, Bahia.

\begin{tabular}{cccc}
\hline Componentes & AutOvalor & Percentagem (\%) & P. Acumulada (\%) \\
\hline 1 & 0,273 & 27,3 & 27,3 \\
2 & 0,171 & 17,1 & 44,4 \\
3 & 0,159 & 15,9 & 60,2 \\
4 & 0,108 & 10,8 & 71,0 \\
\hline
\end{tabular}

Tabela 2 - Autovalor, percentagem da variação total e percentagem acumulada dos quatro primeiros componentes principais, no segundo ciclo de monitoramento no norte da Baía de Todos os Santos, Bahia.

\begin{tabular}{cccc}
\hline COMPONEnTES & AutOVAlor & Percentagem (\%) & P. ACUMulada (\%) \\
\hline 1 & 0,216 & 21,6 & 21,6 \\
2 & 0,209 & 20,9 & 42,4 \\
3 & 0,148 & 14,8 & 57,3 \\
4 & 0,120 & 12,0 & 69,2 \\
\hline
\end{tabular}

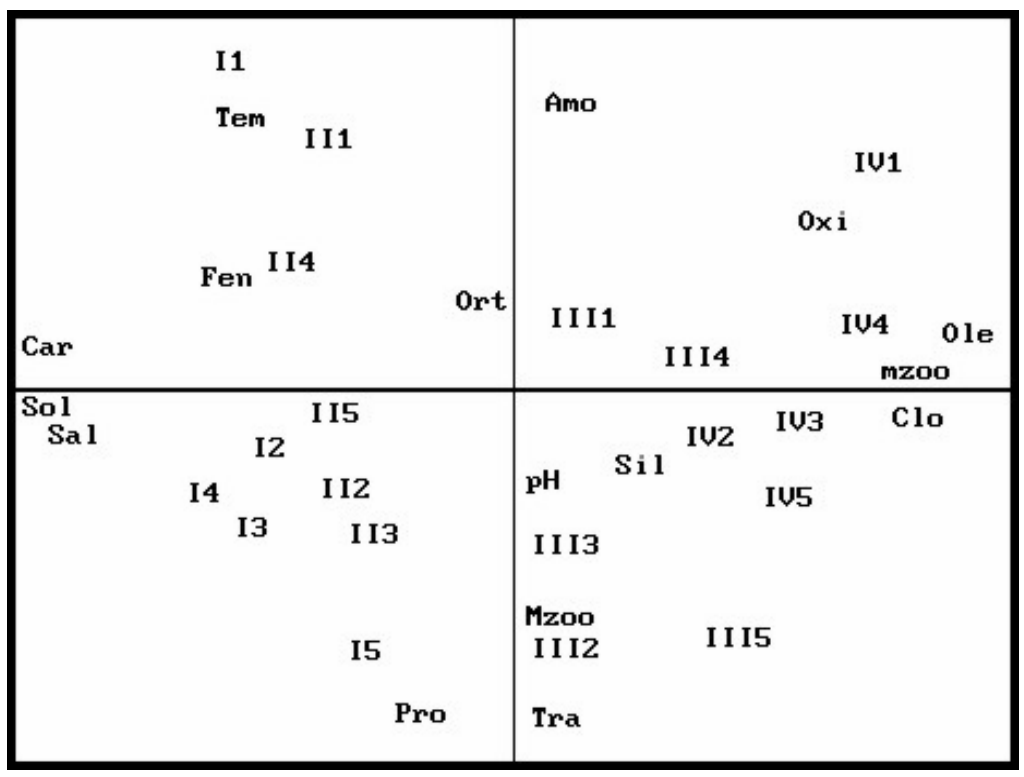

Figura 8 - Diagrama de ordenação com projeção das variáveis oceanográficas e estações de amostragem realizadas nos cruzeiros: I (fevereiro/94), II (maio/94), III (setembro/94), IV (dezembro/94), ao norte da Baía de Todos os Santos (cruzeiros em número romano, estações em número arábico e abreviações das variáveis vide Apêndice I).

Tropical Oceanography, Recife: v. 31, n. 1, p. 31-51, 2003 
Durante o primeiro ciclo (Figura 8), o componente 1 explica 27,3 \% da variância total dos dados. Este fator é influenciado fortemente pelos óleos e graxas $(\mathrm{r}=0,81)$, sólidos dissolvidos totais $(r=-0,79)$, carbono orgânico particulado $(r=-0,79)$, salinidade $(r=-0,74)$, clorofila $(r=0,72)$ e biomassa de microplâncton $(\mathrm{r}=0,72)$. O componente 2 explica 17,1 \% da variância total dos dados, sendo influenciado pelo amônio $(r=0,73)$, transparência $(r=-0,69)$, temperatura $(r=0,69)$ e profundidade $(r=-0,68)$. As variáveis: ortofosfato, $\mathrm{pH}$, mesoplâncton, oxigênio e fenóis foram as menos importantes durante este ciclo.

No segundo ciclo (Figura 9) o componente 1 explica 21,6 \% da variância total dos dados. Este fator é influenciado pela concentração de silicatos ( $r=0,73)$, ortofosfato $(r=0,70)$, sólidos totais em suspensão $(\mathrm{r}=0,72)$ e transparência $(\mathrm{r}=-0,66)$. O componente 2 explicou 20,9 \% da variância dos dados e esteve associado com: temperatura $(\mathrm{r}=0,61)$, e fenóis $(\mathrm{r}=0,70)$. As demais variáveis foram menos importantes durante este período.

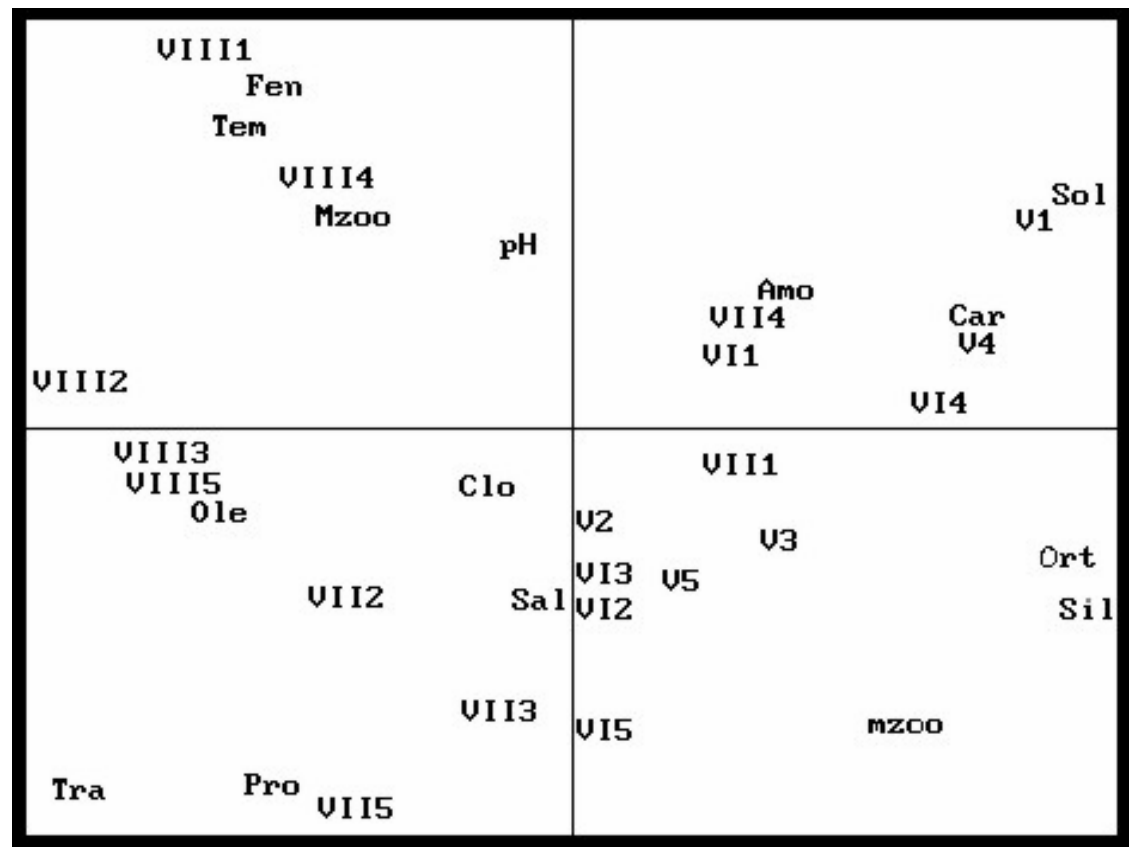

Figura 9 - Diagrama de ordenação com projeção das variáveis oceanográficas e estações de amostragem realizadas nos cruzeiros: V (março/95), VI (junho/95), VII (setembro/95) e VIII (dezembro/95), ao norte da Baía de Todos os Santos (cruzeiros em número romano, estações em número arábico e abreviações das variáveis vide Apêndice I).

Em ambos os ciclos de monitoramento, o agrupamento das estações de amostragem foi conseqüência da variação espacial das condições hidroquímicas, sendo pouco influenciado pelos níveis de biomassa fitoplanctônica (clorofila) e zooplanctônica (micro e meso). Assim, as estações de amostragem 1 e 4 estiveram agrupadas na porção superior do diagrama de ordenação (lado esquerdo e direito), porque foram influenciadas pelas maiores concentrações de amônio e fenóis, carbono orgânico particulado e óleos e graxas oriundos principalmente de processos de extração e 
refino de petróleo, além da temperatura mais elevada, em função da menor profundidade e transparência das suas águas. Já as estações 2, 3 e 5 estiveram localizadas na porção inferior do diagrama de ordenação, porque foram caracterizadas por águas com menor concentração de sólidos em suspensão e por maior profundidade e transparência. Estas estações também não estiveram submetidas diretamente ao efeito físico-químico das atividades de refino e extração de petróleo, uma vez que se encontram mais próximas da área com predomínio das atividades de transporte de petróleo.

\section{CONCLUSÕES}

1. A massa de água tropical presente no norte da Baía de Todos os Santos foi caracterizada como água costeira com pouca influência de aportes fluviais.

2. Os resultados demonstram que existem diferenças significativas, sazonais e espaciais, principalmente nas variáveis hidroquímicas e secundariamente nos níveis de biomassa planctônica.

3. A análise estatística multivariada revelou 2 agrupamentos entre as estações de amostragem; o grupo 1, formado pelas estações sob influência de atividades de refino (estação 1) e extração de petróleo (estação 4). O grupo 2 formado pelas estações sob influência de atividades de transporte de petróleo (estações 2 e 3) e pela estação referência (estação 5).

\section{Agradecimentos}

Os autores reconhecem o financiamento deste projeto através do convênio UFBA/FAPEX/PETROBRÁS. Agradecemos aos técnicos do Instituto de Biologia pelo auxílio na amostragem e processamento das amostras, bem como a Gilson Correa de Carvalho, pela preciosa ajuda na elaboração do mapa. E. M. da Silva integra o Projeto TROCA-WET financiado pela Comissão Européia (ERBIC18CT980264).

\section{REFERÊNCIAS BIBLIOGRÁFICAS}

APHA, American Public Health Association. Standard Methods for the Examination of Water and Wastewater. $18^{\mathrm{a}}$ ed. 1992 ,

DA SILVA, E. M. Programa de Monitoramento dos Ecossistemas ao Norte da Baía de Todos os Santos. Estudos hidrobiológicos. Relatório técnico-científico parcial. Salvador, BA.1994.

DA SILVA, E. M. et al. Ensaio da Disciplina Limnologia e Ecologia de Águas Costeiras do Mestrado em Produção Aquática da UFBA. Instituto de Biologia da UFBA. 1993.

DHN, Diretoria de Hidrografia e Navegação Cartas de Corrente de Maré. Porto de Salvador. Niterói. 1975

CRA, Centro de Recursos Ambientais Bacias Hidrográficas e Saneamento Ambiental no Estado da Bahia. Salvador, BA. 1982.

Tropical Oceanography, Recife: v. 31, n. 1, p. 31-51, 2003 
GARFIELD III, N. The Brazil current at subtropical latitudes. Kingston, 1990, 122p.

Thesis (Doctor of Philosophy) - University of Rhode Island.

FRIEDRICH, H. Marine Biology. Sidgwick \& Jackson, London. 1969, 472 p.

GERMEN, Grupo Ecológico. Baía de Todos os Santos. Diagnóstico Sócio-Ambiental e Subsídios para a Gestão.Salvador, Bahia. 1997.

GRASSHOFF, K. Methods of Seawater Analysis. Verlag Chemie, Weinheim, 1975, 317p.

GUZMAN, J. S.; NUNES FILHO, J. Estudo Hidrográfico para o Gasoduto Itaparica-Aratu. PETROBRÁS, Salvador, BA. 1997

KRAMER, D., KALIN, M. J., STEVENS, E. G., THAIKILL, J. R.; ZWEIFEEL, J. R. Collecting and processing data on fish eggs and larvae in the California Current region. U.S. Dept. of Commerce, NOAA Tech. Rept, NMFS Circ., v. 370, p. 1-38. 1972

LEGENDRE, L.; LEGENDRE, P. Ecologie Numérique. 1. Le traitement multiple des données écologiques. Smasson Presses de L’Université du Quebec, Quebec, 1984, 335 p.

MAFALDA Jr., P. O. Programa de Monitoramento dos Ecossistemas ao Norte da Baía de Todos os Santos. Pesquisa de índices ecotoxicológicos em associações planctônicas.Relatório técnico-científico final. Salvador, BA. 1995.

MAFALDA Jr., P. O. Distribuição e Abundância do Ictioplâncton da Costa Norte da Bahia e suas relações com as Condições Oceanográficas. Rio Grande, 135 p. Tese (Doutorado) Universidade Federal do Rio Grande. 2000.

MARGALEF, R. Ecologia. Ediciones Omega, Barcelona, 1989, 951 p.

SEI, Superintendência de Estudos Econômicos e Sociais da Bahia.. Balanço hídrico do Estado da Bahia. Salvador, BA. 1999, 249p.

STRICKLAND, J. D.; PARSONS, T. R. A Manual of Seawater Analysis. Bull. Fish. Res. Board. Can, v. 125, p. 1-205, 1965.

TER BRAAK, C. J. F. CANOOCO. A Fortran program for canonical community ordination by partial, detrended, canonical analysis, principal component analysis and redundance analysis (version 2.1). ITI-TNO, Wagenigen, 1988, 95p.

THONSEN,H. Massas de água características del Oceano Atlântico, parte sudoeste.

Serviços de Hidrografia Naval H632:1-22. 1962.

TUNDISI, J.; TEIXEIRA, C.; MATSUMURA, T.; KUTNER, M. B.; KINOSHITA, L. Plankton studies in a mangrove environment. IX. Comparative investigations

with coastal oligotrophic waters. Revista Brasileira de Biologia., v. 38, n. 2, p. 301-320. 1978.

WETZAL, R. G. Limnology. Saunders College Publishing, Philadelphia, EUA, 1983, 767p.

Tropical Oceanography, Recife: v. 31, n. 1, p. 31-51, 2003 
ZAR, J. H. Biostatistical Analysis. Prentice Hall, New York., 1984.

Apêndice I - Abreviações das variáveis bióticas e abióticas das figuras 8 e 9.

\author{
Amo - Amônia \\ Car - Carbono orgânico particulado \\ Clo - Clorofila $a$ \\ Fen - Fenóis \\ Ole - Óleos e graxas \\ Ort - Ortofosfato \\ Oxi - Saturação de oxigênio \\ $\mathrm{pH}-\mathrm{pH}$
}

Pro - Profundidade

Sal - Salinidade

Sil - Silicato

Sol - Sólidos totais em suspensão

Tem - Temperatura de superfície

Tra - Transparência

Mzoo - Biomassa de mesozooplâncton

mzoo - Biomassa de microzooplâncton 
Tropical Oceanography, Recife: v. 29, n. 2, 2002 